\title{
Competências de sustentabilidade no âmbito das universidades: revisão sistemática
}

\section{da literatura}

\author{
Sustainability competencies within the universities: systematic literature review \\ Competencias de sostenibilidad dentro de las universidades: revisión sistemática de la literatura
}

Recebido: 05/10/2021 | Revisado: 12/10/2021 | Aceito: 15/10/2021 | Publicado: 18/10/2021

\author{
Jandmara de Oliveira Lima Lira \\ ORCID: https://orcid.org/0000-0002-0046-0424 \\ Universidade Federal de Campina Grande, Brasil \\ E-mail: jandmaralima@gmail.com \\ Maria de Fátima Martins \\ ORCID: https://orcid.org/0000-0002-9578-9555 \\ Universidade Federal de Campina Grande, Brasil \\ E-mail: fatimamartins2005@gmail.com
}

\begin{abstract}
Resumo
As universidades possuem reconhecido poder transformador da sociedade. Por esta razão têm sido consideradas atores-chave no alcance do desenvolvimento sustentável. A educação recebeu destaque na Agenda 2030, ocasionando o aumento de pesquisas relacionadas ao papel das Instituições de Ensino Superior neste novo cenário. O presente trabalho busca compreender como está sendo delineada a pesquisa científica internacional sobre competências de sustentabilidade no âmbito das universidades. Para o alcance deste objetivo, como metodologia, foi realizado um mapeamento bibliométrico, seguido de uma revisão sistemática da literatura. Como fonte de dados foi utilizada a plataforma da Scopus. Os resultados mostram predominância dos estudos na perspectiva acadêmica e foco no desenvolvimento de competências de sustentabilidade direcionadas aos estudantes, uma vez que são considerados os profissionais que tomarão as decisões futuras. Concluiu-se que a Gestão Sustentável no contexto das universidades, sobretudo com ênfase no desenvolvimento de competências, ainda tem sido um campo pouco explorado pelos pesquisadores, embora estas instituições sejam reconhecidas como catalisadoras e atores-chave para o alcance do desenvolvimento sustentável.
\end{abstract}

Palavras-chave: Instituições de ensino superior; Sustentabilidade; Desenvolvimento de competências.

\begin{abstract}
It's a well-known fact that universities have the power to change society. For this reason they are considered key actors of sustainable development. Education was highlighted in the 2030 Agenda increasing the number of papers pertaining to universities' role in this pursuit. This paper aims to understand how international research is being developed on the matter of sustainability in universities. For that, our methodology of choice was a bibliometric mapping followed by a systematic revision of published material on the topic. Our source for data was the Scopus platform.Results show us that the studies are mostly being developed from an academic perspective with sustainable competences aiming towards the students who are seen as the future professionals who will be making important decisions. From that we conclude that sustainable management of universities particularly focusing on development sustainable competencies is a research field that hasn't been fully explored yet even though these institutions are recognized as catalysts for change and understood as having a key role on sustainable development.
\end{abstract}

Keywords: Universities; Sustainability; Competence development.

\section{Resumen}

Es reconocida la influencia transformadora de las universidades en la sociedad. Por esta razón, han sido consideradas actores clave en el alcance del desarrollo sustentable. La educación recibió destaque en la Agenda 2030, generando el aumento de investigaciones relacionadas al rol de las instituciones de Educación Superior en este nuevo escenario. El presente trabajo, pretende comprender cómo está siendo delineada la pesquisa científica internacional sobre capacidades de sustentabilidad en el ámbito de las universidades. Para lograr tal objetivo, como metodología fue realizado un mapeo bibliométrico, seguido de una revisión sistemática. Como fuente de datos, fue utilizada la plataforma Scopus. Los resultados muestran predominancia de los estudios en la perspectiva académica y foco en el desarrollo de capacidades de sustentabilidad direccionadas a los estudiantes, una vez que son considerados los profesionales que tomarán decisiones futuras. Se concluye que la Gestión Sostenible en el contexto de las universidades, principalmente con énfasis en el desarrollo de capacidades, todavía ha sido un campo poco explorado 
por los investigadores, aunque estas instituciones son reconocidas como catalizadoras y actores clave para el alcance del desarrollo sustentable.

Palabras clave: Instituciones de educación superior; Sostenibilidad; Desarrollo de habilidades.

\section{Introdução}

Apesar da indefinição do termo desenvolvimento sustentável, tem sido amplamente aceito o conceito divulgado pela Comissão do Meio Ambiente e Desenvolvimento (1987), publicado por meio do Relatório de Brundtland, que o reconhece como "o desenvolvimento que satisfaz as necessidades do presente sem comprometer a capacidade das gerações futuras de satisfazerem as suas próprias necessidades" (WCED, 1987, p. 43). Este permanece sendo o conceito mais popular e difundido. Desde a década de 70, a Organização das Nações Unidas realiza encontros internacionais para tratar deste tema. Segundo Berchin at al (2018, p.770), estes espaços são importantes em razão da persistência desta indefinição conceitual.

Em 2015, na cidade de Nova York, aconteceu o evento que deu origem aos Objetivos de Desenvolvimento Sustentável (ODS). São 17 ao todo, compostos por 169 metas complexas, ambiciosas e com uma estrutura de monitoramento por meio de relatórios anuais a ONU, além e indicadores associados (Valência et al, 2019, p.5). Consiste em uma ampliação da estrutura dos Objetivos de Desenvolvimento do Milênio, estabelecidos em 2000 para serem alcançados até 2015 (Litre et al, 2020, p.3). A finalidade dos ODS é conciliar crescimento econômico e sustentabilidade do sistema, com transformações profundas na forma de gerenciamento das organizações e nos seus processos de produção (Bastida et al, 2020). Sendo organizações com elevado potencial de transformação social, as universidades tem sido consideradas catalizadores na implantação dos ODS. Segundo Lozano at (2013, p.11), já na Conferência de Estocolmo, em 1972, a educação foi reconhecida, formalmente e em nível internacional, na promoção da proteção e conservação ambiental

As universidades não são apenas provedoras de conhecimento, mas também atores-chave e de relevância política na sociedade, portanto, devem atuar como modelos pioneiros em nome do desenvolvimento sustentável e levar a sério o seu papel (Schopp, Bornemann \& Potthast, 2020). As universidades públicas, sobretudo, têm o dever de implantar uma sustentabilidade mais profunda, pois sua finalidade é atender aos interesses da sociedade (Baker-Shelley, Zeijl-Rozema \& Martens, 2017, p.263). Deste modo, as universidades comprometidas desempenham um papel decisivo para garantir que o contexto socioeconômico em que atuam sigam na direção do desenvolvimento sustentável (Di Nauta at al, 2020, p.16).

Diante do cenário atual, o grande desafio é encontrar a equação que permita equilibrar a dinâmica econômica, social e ambiental em nível global, com a participação do governo, organizações e sociedade em geral. Tem crescido o interesse dos pesquisadores em direção a participação das universidades como disseminadoras da sustentabilidade em diferentes níveis, seja na perspectiva do ensino, como formadora dos futuros profissionais, e assim da capacitação do docente como agente de transformação e, consequentemente, na capacitação do aluno, preparando-o para um mercado de trabalho cada vez mais lincado com esta nova realidade. Ressaltam Avery e Nordén (2017) que se a educação para a sustentabilidade visa, em última análise, possibilitar a mudança, então as condições estruturais para a mudança precisam ser abordadas, começando com as Instituições de Ensino Superior que impulsionam o desenvolvimento de conhecimentos e capacidades.

Ainda que o progresso limitado da integração do Desenvolvimento sustentável aos currículos universitários (Leal Filho at al, 2018, p. 287), é perceptível a ênfase das pesquisas realizadas em direção, sobretudo, ao campo acadêmico, seja na formação de professores, seja na reconfiguração curricular ou mesmo na atualização das abordagens pedagógicas ou metodológicas. E quanto a gestão universitária? O ambiente acadêmico é coerente com a explanação e desenvolvimento de conhecimentos, projetos e pesquisas sobre a sustentabilidade? A vinculação entre educação e experiências reais precisa assegurar que as instituições de ensino superior estejam alinhadas com o conhecimento que disseminam.

A gestão por competências pode representar uma importante ferramenta estratégica nesse processo, no sentido de capacitar toda comunidade universitária para cooperação com os princípios do desenvolvimento sustentável. Até porque 
competências organizacionais são adquiridas a partir de uma construção coletiva, por meio do trabalho sinérgico, influenciado por competências individuais (Zarifian, 2008; Le Boterf, 2003).

O conceito de competências tem sido amplamente aceito como o conjunto de conhecimentos, habilidades e atitudes (CHA) no campo individual. Munck at al (2011, p.109) descrevem duas dimensões que compõe as competências: os imputs algo que o indivíduo necessita ter para um desempenho competente, como conhecimento, julgamento, habilidade ou força - e os outputs, a efetiva aplicação dessas capacidades para lidar com qualquer assunto.

Assim sendo, este trabalho busca compreender como está sendo delineada a pesquisa científica internacional sobre competências de sustentabilidade em Instituições de Ensino Superior. Para isto, foi realizado um mapeamento bibliométrico, seguido de uma revisão sistemática da literatura, por meio dos quais buscou-se identificar quais as lacunas existentes, a fim de contribuir com novas pesquisas que aprofundem a temática e possam colaborar com a implantação do desenvolvimento sustentável.

\section{O Desenvolvimento de Competências Incorporado a Gestão Universitária Sustentável}

As universidades podem ser os principais atores para apoiar o desenvolvimento sustentável (DS) a partir de estratégias aplicadas a pesquisa e educação das gerações futuras, mas também por meio da implementação da sustentabilidade em suas organizações (Hauff \& Nguyen, 2014). Ainda que seja perceptível a estrutura multifacetada de uma universidade e conhecido seus importantes papéis para sociedade externa e comunidade interna, os estudos realizados em instituições de ensino superior com foco no desenvolvimento sustentável são predominantemente voltados para a educação. Neste sentido, a literatura tem disponibilizado importantes contribuições.

Brundiers, Wiek e Redman (2010), por exemplo, apresentam uma análise das estruturas de competências-chave de sustentabilidade que podem ser adquiridas pelos alunos a partir de experiencias reais. Propõem um modelo funcional e progressivo a ser integrado ao currículo de graduação e, ainda, sugerem estratégias para a sua implementação. Wals (2014) indica a necessidade de diferenciar competência de desenvolvimento sustentável (relativas à prática do cidadão para contribuir com uma vida sustentável, tanto profissionalmente, quanto pessoalmente) e competência de Educação para o desenvolvimento sustentável (relacionadas a capacidade do educador em ajudar as pessoas a desenvolverem competência DS por meio de práticas inovadoras de ensino e aprendizagem).

A atuação das universidades não se concentra apenas na educação. Elas conduzem também tarefas administrativas e podem contribuir para o desenvolvimento sustentável em contextos variados. Elas também disseminam os resultados do que produzem para a sociedade, contando com uma equipe universitária para trabalhar em diversas áreas que podem precisar de outras competências além das necessárias para o campo da educação (Schopp, Bornemann \& Potthast, 2020). As iniciativas de sustentabilidade podem ser promovidas por diferentes atores-chave, em diferentes ambientes e com diferentes formações, como: engenheiros, economistas, ambientalistas, advogados e profissionais de saúde e outros (Velazquez, 2006). A partir de uma abordagem integrada, uma universidade sustentável deve atuar de forma sustentável em todas as áreas, balizadas pelas dimensões econômica, ecológica e social (Hauff \& Nguyen, 2014).

Velazquez (2006) propôs um modelo para auxiliar líderes universitário na implantação da sustentabilidade em uma perspectiva mais holística. O modelo é composto por quatro fases: desenvolvimento de uma visão de sustentabilidade para a universidade, incorporação da sustentabilidade a missão, estabelecimento de um comitê de sustentabilidade e implantação de estratégias de sustentabilidade.

Ainda assim, anos mais tarde o que se observa é que a orientação da universidade como um todo tende a ser comunicada por meio de indicadores descontextualizados e estruturada por questões financeiras. Isto inclui os níveis departamentais, onde não existe espaço para aspectos mais complexos e as energias são direcionadas para tarefas fragmentadas 
específicas, incentivadas com base em financiamentos e avaliações. Os processos de reflexão implicam no engajamento mais ativo tanto das atividades de ensino e aprendizagem, como na reformulação contínua das estruturas universitárias (Avery \& Nordén, 2017).

O corpo docente pode ser considerado um catalizador do desenvolvimento sustentável relacionado ao ensino. O que pode estender a instituição de modo geral, seja nas ações de divulgação ou no exercício de funções de gestão (Barth \& Rieckmann, 2012). Neste sentido, para além do foco exclusivo no campo acadêmico, a formulação de competências com objetivos de aprendizagem pode ser usada como uma forma de garantir que as questões de sustentabilidade sejam incorporadas as práticas de ensino, mas também a universidade em sua totalidade (Avery \& Nordén, 2017).

O conceito de competências de maneira ampla deve considerar não apenas o indivíduo isolado, mas o plano coletivo, com enfoque no agrupamento de conhecimentos, habilidades e atitudes que são estabelecidos por meio da sinergia do grupo (Zarifian, 2008; Le Boterf, 2003). Ofei-Manu e Didham (2018, p. 1179) definem competências de sustentabilidade como as "capacidades necessárias, como uma variedade de diferentes conjuntos de conhecimentos e habilidades, juntamente com valores e certas questões éticas de que as pessoas precisam para poder contribuir para o desenvolvimento sustentável".

A comunicação de conhecimentos e competências para o Desenvolvimento Sustentável não pode ser vista como um processo puramente de cima para baixo, é crucial envolver igualmente todos os atores (Schopp, Bornemann \& Potthast, 2020), o que inclui não apenas o ensino, mas a administração e participação dos stakeholders.

\section{Metodologia}

Esta pesquisa corresponde a um mapeamento bibliométrico, seguido de uma revisão sistemática da literatura, a fim de compreender a evolução dos estudos realizados com a temática desenvolvimento de competências de sustentabilidade em universidades e identificar as principais lacunas existentes. Trata-se de uma análise exploratória e descritiva. A bibliometria permite ao pesquisador conhecer os principais autores e trabalhos publicados em uma determinada área do conhecimento, a partir do uso de parâmetros como citação, referências bibliográficas, levantamento dos periódicos, parcerias de coautorias e impactos em outros estudos.

A escolha da revisão sistemática da literatura se justifica por ser um método mais confiável de análise, por permitir o aprofundamento dos temas desenvolvidos e dos principais aspectos explorados nas pesquisas, além de identificar os gaps no campo de pesquisa. Segundo Faria (2016), corresponde a uma metodologia de pesquisa com rigor cientifica, cujo objetivo é minimizar o enviesamento do estado da arte, pois é realizada uma recolha exaustiva de textos publicados sobre um tema e, neste processo, são descritos e justificados todos os procedimentos adotados. Segundo o autor, a credibilidade deste tipo de pesquisa esta no como se estabelece as regras. Por esta razão, os critérios devem ser bem definidos no intuito de atribuir validade cientifica as obras que forem selecionadas para análise.

Os softwares existentes para análises de pesquisas apresentaram-se insuficientes para o alcance do que esta pesquisa pretendia. Em razão disto, foi adotado o critério de analise manual. A Figura 1 apresenta as principais etapas seguidas na sistematização da literatura a partir do mapeamento da produção cientifica internacional: 
Figura 1 - Etapas da pesquisa.

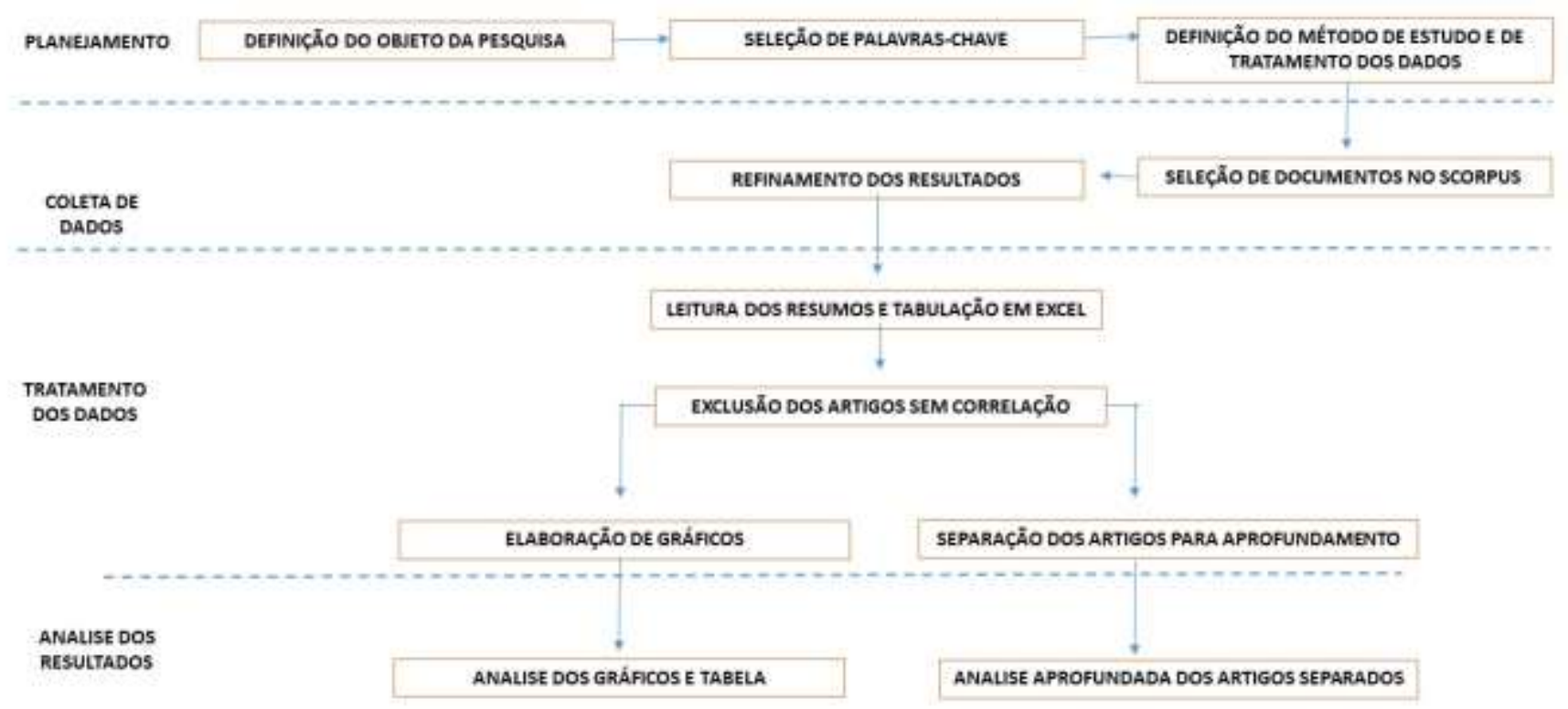

Fonte: Autores.

A primeira etapa corresponde a delimitação do estudo. O tema e objetivo surgiram da necessidade em conhecer o quadro evolutivo de pesquisas em uma temática específica, neste caso relativo ao desenvolvimento de competências de sustentabilidade na esfera universitária, o que conduziu a escolha de três termos para aplicação na base de dados: "university", "competence" e "sustainable development".

A segunda etapa foi a obtenção dos dados, realizada em 22 de outubro de 2020, às 14 horas e 40 minutos. Para tal foi utilizada a base Scopus, da Elsevier. A escolha justifica-se em virtude do scopus ser uma base de dados bibliográficos que reúne uma extensa variedade de títulos e editoras internacionais e, ainda por indexar um maior volume de publicações com a temática pesquisada em relação a plataforma Web of Science. A partir da aplicação dos termos acima citados, a Web of Science filtrou 101publicações, enquanto que o Scopus apresentou 326 documentos.

As palavras "university", "competence" e "sustainable development" foram aplicadas entre aspas e em conjunto com a expressão "AND" para obtenção de documentos que possuíssem os três termos em seu corpo (título, resumo ou palavrachave). As classes temáticas foram limitadas a três áreas específicas: ciências sociais; ciências ambientais; e negócios, gestão e contabilidade. Não foi aplicado refinamento quanto aos anos com ocorrência de publicação, sendo considerado todo período apresentado, a fim de se estabelecer uma compreensão histórico-evolutiva ao longo do tempo, o que abrangeu o período de 1995 até a data da pesquisa, incluindo documento já aprovados para 2021, tendo como parâmetro os adicionados na Scorpus até os últimos 7 dias da data de filtragem dos dados. Artigo foi o tipo de documento selecionado, obtendo um total de 128 pesquisas.

Também foi realizado um filtro com base nas palavras-chave utilizadas nos documentos, o que totalizou 110 publicações. As palavras-chave foram escolhidas de acordo com quatro temas, conforme apresentado no Quadro 1: 
Quadro 1 - Palavras-chave escolhidas para o mapeamento bibliométrico.

\begin{tabular}{|c|c|}
\hline TEMAS & PALAVRAS-CHAVE DO DOCUMENTO \\
\hline SUSTENTABILIDADE & $\begin{array}{l}\text { sustentabilidade - desenvolvimento sustentável - metas do desenvolvimento sustentável - gestão ambiental } \\
\text { - gestão - SDG - Ciência da Sustentabilidade - Objetivo do Desenvolvimento Sustentável }\end{array}$ \\
\hline COMPETÊNCIA & $\begin{array}{l}\text { competências - competência profissional - desenvolvimento de competência - competência intercultural - } \\
\text { competência de ação - competência antecipatória - competências básicas - estrutura de competências } \\
\text { C2P2S - capacidades }\end{array}$ \\
\hline UNIVERSIDADE & $\begin{array}{l}\text { setor universitário - universidade - instituições de ensino superior - universidades - ensino superior - ensino } \\
\text { superior para o desenvolvimento sustentável }\end{array}$ \\
\hline $\begin{array}{ll}\text { APRENDIZADO } & \text { E } \\
\text { DESENVOLVIMENTO } & \end{array}$ & $\begin{array}{l}\text { aprendendo - desenvolvimento profissional - treinamento de professor - capacitação - treinamento - } \\
\text { aprendizagem experiencial - aprendizado ativo - aprendizagem baseada em problemas - ensinando e } \\
\text { aprendendo - transformando ensino e aprendizagem - educação para sustentabilidade - coprodução de } \\
\text { conhecimento - objetivos de aprendizado - aprendizado hibrido - educação para o desenvolvimento } \\
\text { sustentável -educação ambiental - educação para a sustentabilidade }\end{array}$ \\
\hline
\end{tabular}

Fonte: Autores.

A terceira etapa está subdividida em dois momentos. Primeiramente, a partir da leitura dos resumos dos artigos, as principais informações (título, ano, autores, principais abordagens) foram sistematizadas em uma planilha de Excel. Foram excluídos da planilha os artigos sem correlação com a pesquisa, ou seja, aqueles cuja pesquisa não foi desenvolvida no âmbito da universidade ou cujo foco principal não é o desenvolvimento de competências de sustentabilidade. Foram excluídos nesta etapa 22 artigos, restando 88.

Com base neste resultado, o estudo foi classificado em duas abordagens: Ensino e Gestão. Para aprofundamento da leitura, na quinta etapa, utilizou-se como critério, a abordagem com menor número de artigos, neste caso, a abordagem de gestão, com apenas quatro pesquisas apresentadas na amostra. A análise foi feita com base nas perspectivas de ensino e de gestão. Na perspectiva do ensino a análise foi realizada de modo mais amplo, a partir dos resumos e tabulação. Na perspectiva da gestão, a análise foi realizada a partir da leitura completa do artigo. Optou-se por este critério de análise por ser a gestão o aspecto menos explorado nas pesquisas e por representar, portanto, uma lacuna na literatura. Conhecer o que tem sido estudado na área de gestão sustentável, possibilitara aos pesquisadores compreender quais focos de estudo ainda precisam ser explorados.

\section{Resultados e Discussão}

A pesquisa apresenta, a partir de 2017, um interesse crescente dos pesquisadores em estudos relacionados ao desenvolvimento de competências de sustentabilidade no âmbito das Instituições de ensino superior, havendo uma lacuna de 10 anos sem estudos registrados com a temática, no período de 1996 a 2006, como pode ser visto a seguir: 1995 (0),1996 (1), 2006 (2), 2008 (2), 2009 (6), 2010 (5), 2011 (1), 2012 (4), 2013 (2), 2014 (3), 2015 (5), 2016 (3), 2017 (13), 2018 (14), 2019 (15), 2020 (11). Também conta com uma publicação já referente ao ano de 2021. Dentre os estudos registrados, 83 tem o Ensino como foco principal $(95,4 \%)$ e 4 focam na gestão universitária $(4,6 \%)$. O gráfico a seguir demonstra a quantidade de periódicos publicados em cada ano: 
Gráfico 1 - Número de publicações por ano.

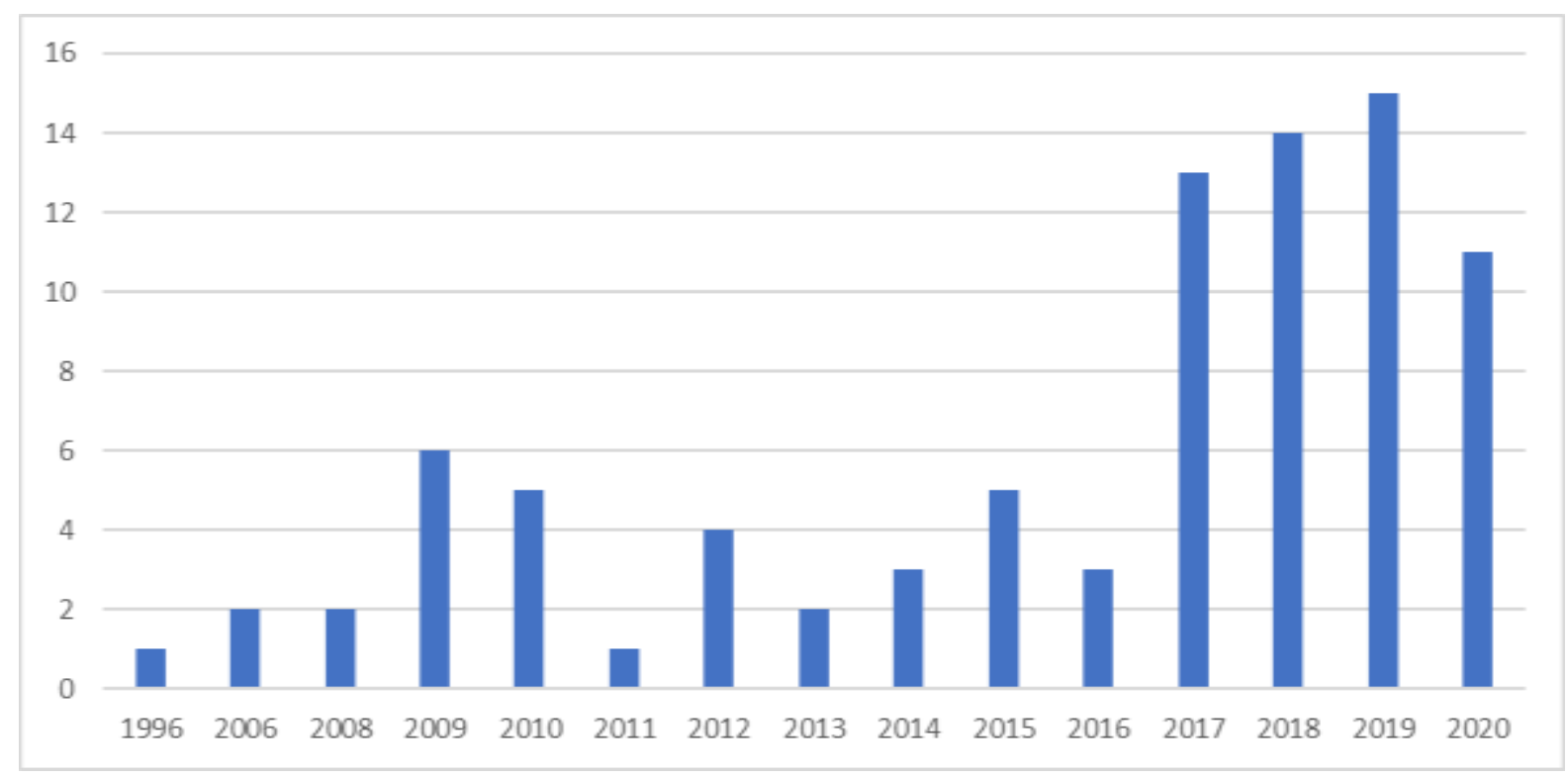

Fonte: Autores.

É possível observar um crescente interesse nos últimos anos. Os periódicos com maior número de publicação apresentados nos resultados são: Sustainability (23), International Journal of Sustainability in Higher Education (22) e Journal of Cleaner Production (17).

$\mathrm{O}$ artigo mais citado tem como título "Real-world learning opportunitirs in sustainability: from classroom into the real world”, publicado em 2010, escrito por Katja Brundiers, Arnim Wiek e Charles L. Redman. Foi citado 248 vezes, publicado no International Journal of Sustainability in Higher Education e tem como objetivo analisar o tipo e a extensão em que as competências-chave de sustentabilidade podem ser adquiridas em oportunidades de aprendizagem no mundo real. Os autores propõem um modelo funcional e progressivo a ser integrado ao currículo universitário, além de sugerir estratégias para sua implementação.

O segundo artigo com maior número de citação (175 citações) foi escrito por Arjen E.J. Wals e publicado em 2014. O artigo tem como título "Sustainability in higher education in the context of the un DESD: A review of learning and institutionalization processes". Está baseado empiricamente em uma revisão da Década da Educação para o Desenvolvimento Sustentável da ONU (UN DESD). Apesar de dirigir uma atenção especial a competências orientadas para a sustentabilidade no ensino superior, apresenta contribuições para universidade como um todo. Deste modo, ele receberá uma análise mais detalhada posteriormente neste estudo.

\subsection{Análise das publicações na perspectiva do ensino}

Os primeiros estudos apresentados nos resultados já introduziram a necessidade de alinhar teoria à prática no ensino da sustentabilidade, abordando a importância de estudos de caso e ligação dos alunos ao "mundo real" e seus problemas complexos. O Quadro a seguir apresenta as principais abordagens tratadas nos estudos realizados entre 1995 até a data da presente pesquisa, ou seja, 21 de outubro de 2020 e cujo foco é o ensino: 
Quadro 2 - principais abordagens tratadas nos estudos realizados (1995-2020).

\section{TÍTULO DA PESQUISA}

Uma questão de conexão: os 4 Cs da aprendizagem na formação inicial de professores para a sustentabilidade. (2021)

Implementação dos ODS no ensino universitário: um curso de desenvolvimento profissional de professores da educação para a sustentabilidade para uma ação transformadora. (2020)

Competências para a resolução de problemas complexos: Um estudo transversal sobre o Transdisciplinaridade ensino superior para a aprendizagem da sustentabilidade e transdisciplinaridade. (2020)

Os objetivos de desenvolvimento sustentável (ODS) como base para habilidades de inovação para engenheiros no contexto da indústria 4.0 (2020)

Como melhorar as competências de sustentabilidade da formação de Experiencia educacional inovadora - ODS 13 professores? Indagando o conhecimento prévio sobre mudanças climáticas em alunos do ensino fundamental. (2020)

Ensinando nativos digitais a adquirir competências para o desenvolvimento sustentável. (2020)

Educação moral para o desenvolvimento sustentável: comparação das percepções de professores universitários na China e no Paquistão. (2020)

Engenheiros mudando o mundo: educação para a sustentabilidade em universidades técnicas romenas - uma análise de conteúdo empírica baseada na web. (2020)

Avaliação do desenvolvimento de competências profissionais em estudantes universitários: Sustentabilidade e jogos sérios. (2020)

Reflexões para a promoção de competências na educação para o desenvolvimento sustentável usando o exemplo das mudanças climáticas. (2020)

Construindo competência interpessoal em estudantes de arquitetura e design urbano por meio de cidades inteligentes em uma instituição de ensino superior. (2019)

A avaliação dos atributos de sustentabilidade da graduação no local de trabalho: vantagens potenciais do uso da teoria do comportamento planejado (TPB). (2019)

O 'lado lunar' da história: explorando a sustentabilidade dos estágios curriculares no ensino superior. (2019)

Ciência à luz da rua e a medida de competência global da OCDE: um novo parâmetro para a internacionalização? (2019)

Rumo à integração dos ODS no ensino de planejamento superior: percepções do programa de estudos de urbanismo integrado em Belgrado. (2019)

Ensinando para um mundo melhor. Sustentabilidade e Objetivos de Desenvolvimento Sustentável na construção de uma universidade transformadora. (2019)

Variáveis que influenciam a formação inicial de professores em educação para o desenvolvimento sustentável: um estudo de caso de duas universidades espanholas. (2019)

$\mathrm{O}$ que os estudantes universitários sabem sobre as metas de desenvolvimento sustentável? Uma abordagem realista para a recepção deste programa da ONU entre a população jovem. (2019)

Educação em engenharia e desenvolvimento de competências para a sustentabilidade. (2019)

Implementando a orientação por competências: Rumo a uma educação alinhada de forma construtiva para o desenvolvimento sustentável no ensino e aprendizagem de nível universitário. (2019)

Educação impactante em engenharia por meio de colaborações de energia sustentável com entidades públicas e privadas. (2019)

Concepção de um programa de mestrado em energia solar: Infraestrutura, estrutura e resultados. (2019)

\section{ABORDAGEM}

Capacitação docente

Metodologias ativas- capacitação - ODS

ODS - Inovação

Metodologias de ensino - inovação dirigida a nativos digitais

Educação moral - práticas e crenças

configuração curricular

metodologias ativas - jogos

Objetivos do Desenvolvimento Sustentável - uso de mídias digitais

Cidades inteligentes - competências interpessoais

Teoria do Comportamento planejado desenvolvimento de ferramentas de avaliação

estágios curriculares como ferramenta para o alcance dos ODS

ODS- Desenvolvimento de competências globais Internacionalização das universidades

ODS - compatibilidade curricular e urbanismo

ODS - conceito para sustentabilidade

competências ambientais e varáveis demográficas e educacionais

Objetivos do Desenvolvimento Sustentável

Competências para resolução de conflitos ligados a sustentabilidade

orientação metodológica - o modelo de árvore da ciência

aprendizagem ativa multidisciplinar e parcerias energéticas sustentáveis

Energias renováveis - desenvolvimento de: competências cognitivas, habilidades práticas e aplicadas 
Cursos online abertos massivos na formação inicial de professores de ciências sociais: experiências, concepções metodológicas e uso tecnológico para o desenvolvimento sustentável. (2019)

Educação para o desenvolvimento sustentável no contexto da educação superior na Bolívia. Percepções de professores universitários. (2019)

Oportunidades de qualificação em comunicação ambiental em unidades de conservação. (2019)

Educação para o Desenvolvimento Sustentável em uma Universidade Suíça: Navegando nas Armadilhas da Institucionalização. (2018)

Formação de professores do ensino médio sob o prisma da sustentabilidade: o caso da Universitat de València. (2018)

Identificação dos níveis de consciência sustentável dos professores em formação por meio de um e-portfólio. (2018)

Transferência de soluções de sustentabilidade entre contextos por meio de parcerias cidade-universidade. (2018)

Orientando o desenvolvimento sustentável no ensino superior - Resultados do Brasil e da Finlândia. (2018)

O pensamento sistêmico no âmbito da educação para o desenvolvimento sustentável (EDS) - um modelo de competência heurística como base para a formação de professores (ciências). (2018)

Compromisso de saúde global: em casa e no exterior. (2018) uso das tecnologias de informação e comunicação (TIC) e cumprimento dos ODS

processo ensino-aprendizagem: operacionalização, conceituação, implementação e institucionalização programas de treinamento e comunicação ambiental em áreas protegidas

políticas e abordagens para a gestão de mudanças necessárias para compreensão de armadilhas

ODS e competências para educação, ciência e cultura

desenvolvimento da reflexão e pensamento crítico para consciência sustentável

parceria cidade-universidade entre países

política nacional de orientação do Desenvolvimento Sustentável e na abordagem pedagógica na IES

Pensamento sistêmico

Metodologias de ensino - pensamento sistêmico e consciência global

Transdisciplinaridade no ensino superior para a sustentabilidade: como os discursos são Transdisciplinaridade

abordados no ensino de engenharia. (2018)

Infundindo sustentabilidade na educação de engenharia de software: Lições aprendidas com projetos fundamentais. (2018)

Competência ecológica de bacharéis de perfil socioeconômico no contexto dos princípios da educação para o desenvolvimento sustentável. (2018)

Implementar as metas de desenvolvimento sustentável em nível universitário. (2018)

Educação para o desenvolvimento sustentável hoje: uma área problemática para superar as dificuldades de adaptação pedagógica (a exemplo de uma escola superior). (2018)

Avaliando as competências ambientais de professores em formação inicial na Espanha: um estudo comparativo entre duas universidades. (2018)

Participação de alunos autores em relatórios de sustentabilidade. (2018)

Conectando competências e abordagens pedagógicas para o desenvolvimento sustentável no ensino superior: uma revisão da literatura e proposta de estrutura. (2017)

Ensine-o a sustentá-lo! Atitudes ambientais de alunos húngaros em treinamento de professores na Sérvia. (2017)

Caminhos para a produção mais limpa nas Américas I: ponte entre as lacunas indústriaacademia na transição para a sustentabilidade. (2017)

O seminário virtual europeu sobre desenvolvimento sustentável como uma oportunidade para o desenvolvimento de competências da equipe ESD dentro dos currículos universitários. (2017)

Catalisando Mudança no Ensino Superior para o Desenvolvimento Sustentável: Uma revisão das iniciativas de desenvolvimento profissional para educadores universitários. (2017)

Sessões de treinamento que promovem a colaboração transdisciplinar para o desenvolvimento sustentável: estudos de caso da Albânia e Kosovo. (2017)

Desenvolvimento profissional de competências de sustentabilidade no ensino superior: o papel do empoderamento. (2017)
TIC e serviços de software para infundir hábitos sustentáveis

desenvolvimento de competência ecológica

Objetivos do Desenvolvimento Sustentável no currículo Universitário

Áreas problemáticas na adaptação pedagógica

Reconfiguração curricular

Relatório Anual de Sustentabilidade - participação dos alunos

abordagens pedagógicas - educação holística e sistêmica para futuros lideres

consciência ambiental de professores

parceria indústria- academia (produção mais limpa)

Seminário Virtual Europeu sobre Desenvolvimento Sustentável

quadros de políticas internacionais e revisão da literatura

colaboração ciência sociedade em sessões de treinamento

Desenvolvimento organizacional, desenvolvimento profissional e empoderamento 
Desenvolvimento profissional de ESD de educadores universitários na Sérvia, Croácia e Macedônia: uma análise comparativa. (2017)

Aprender para e sobre a sustentabilidade no ensino superior - uma perspectiva regional baseada em experiências do Báltico e do Mediterrâneo. (2017)

Princípios de EDS no ensino superior na perspectiva dos países da Europa Central e Oriental. (2017)

Aspectos ecológicos no enfoque da formação profissional: discurso substantivo e metodológico. (2017)

Reorientando os currículos de desenvolvimento pedagógico e profissional do ensino superior em direção à sustentabilidade - uma perspectiva romena. (2017)

Planejamento regional, metas de sustentabilidade e combinação entre a prática educacional e os planos de clima, energia e negócios. (2016)

Redes de dados e educação para a sustentabilidade em universidades africanas: um estudo de caso para a África Subsaariana. (2016) desenvolvimento de competências para sustentabilidade - oportunidades, barreiras e motivadores

rede de ensino em educação para o desenvolvimento sustentável e esforços coletivos entre países

parcerias de universidades europeias e os princípios da educação para sustentabilidade

competências eco-profissionais

Reconfiguração curricular

Planejamento regional

Integração entre pesquisadores - redes de pesquisa e educação com foco na sustentabilidade, particularmente as mudanças climáticas

Estratégias de educação para o desenvolvimento sustentável - perspectivas dinamarquesas e australianas. (2016)

Proposta de um modelo teórico baseado na competência em um curso de engenharia civil. (2015)

Integração entre instituições incorporado ao ensino

desenvolvimento de competências genéricas na graduação

Pedagogias de preparação: Uso de diários reflexivos na operacionalização e desenvolvimento da competência antecipatória. (2015)

Um intercâmbio educacional virtual: uma aula virtualmente compartilhada norte-sul sobre desenvolvimento sustentável. (2015)

Gamiticação: Estratégias para otimizar o processo de aprendizagem e aquisição de competências em contextos universitários. (2015)

Desenvolvimento de um kit de ferramentas prático para apoiar a integração do ecodesign em programas de engenharia. (2015)

Educando agentes de mudança para a sustentabilidade - Aprendizagem do primeiro mestre em gestão de sustentabilidade em administração de negócios. (2014)

Sustentabilidade na Educação Superior no contexto da ONU DESD: uma revisão dos processos de aprendizagem e institucionalização.

Desenvolvimento Sustentável como Desafio para Estudantes de Graduação: O Módulo "A Ciência Carrega Responsabilidade" no Programa de Bacharelado da Leuphana: Comentário sobre "Um Estudo de Caso de Ensino de Responsabilidade Social para Estudantes de Doutorado em Ciências do Clima". (2013)

Mudança das demandas profissionais no desenvolvimento regional sustentável: um competências transfronteiriças processo de desenho de currículo para atender às competências transfronteiriças. (2013)

Abraçando o paradigma de aprendizagem para promover o pensamento sistêmico. (2012)

Estamos treinando engenheiros para a sustentabilidade? Comparação entre as competências obtidas e as necessidades da indústria sueca. (2012)

Coprodução de conhecimento em teses de doutorado transdisciplinares sobre o Transdisciplinaridade

desenvolvimento da paisagem - Uma análise dos papéis dos atores e dos tipos de conhecimento nas diferentes fases da pesquisa. (2012)

Integrando o desenvolvimento sustentável em cursos de gestão de operações (2011)

Capacidades dos professores para trabalhar em prol da paz e do desenvolvimento sustentável. (2010) desenvolvimento de competências antecipatórias

Incorporação de experiências internacionais e interculturais

Teoria e prática desenvolvimento de competências genéricas essenciais.

Ecodesign no ensino com foco na sustentabilidade

Gestão de sustentabilidade - capacitação de gerentes externos a universidade

Revisão da Década da Educação para o Desenvolvimento Sustentável da ONU

modelos inovadores de ensino e da inter e transdisciplinaridade

Avaliação da competência do pensamento sistêmico Competências para tomada de decisão

Cursos de treinamento

capacitação docente e reformas educacionais 


\begin{tabular}{|c|c|}
\hline Sustentabilidade, ciência e ensino superior: A necessidade de novos paradigmas. (2010) & Aprendizagem baseada em problemas \\
\hline $\begin{array}{l}\text { Oportunidades de aprendizagem do mundo real em sustentabilidade: da sala de aula } \\
\text { para o mundo real. (2010) }\end{array}$ & $\begin{array}{l}\text { Teoria e prática - estudos de caso. Aprendizagem } \\
\text { organizacional }\end{array}$ \\
\hline $\begin{array}{l}\text { O programa de pós-graduação em ciências ambientais da Universidade Americana de } \\
\text { Beirute: uma iniciativa de ESD no mundo árabe. (2010) }\end{array}$ & $\begin{array}{l}\text { Aprendizagem baseada em competências - } \\
\text { pensamento sistêmico }\end{array}$ \\
\hline $\begin{array}{l}\text { O que os alunos de engenharia aprendem nos cursos de sustentabilidade? O efeito da } \\
\text { abordagem pedagógica. (2010) }\end{array}$ & $\begin{array}{lllll}\begin{array}{l}\text { Abordagem pedagógica } \\
\text { desenvolvimento sustentável }\end{array} & \text { de } & \text { cursos } & \text { de } \\
\end{array}$ \\
\hline $\begin{array}{l}\text { Desenvolvimento do modelo de aprendizagem para o desenvolvimento sustentável no } \\
\text { ensino superior. (2009) }\end{array}$ & $\begin{array}{l}\text { Teoria e prática por meio de estudos de caso - } \\
\text { modelos de aprendizagem para o desenvolvimento } \\
\text { sustentável }\end{array}$ \\
\hline $\begin{array}{l}\text { Educação em competências básicas para o desenvolvimento sustentável. O papel da } \\
\text { universidade. (2009) }\end{array}$ & Teoria, prática e ética para sustentabilidade \\
\hline $\begin{array}{l}\text { Sobre os objetivos de laboratórios de instrução, avaliação individual e uso de } \\
\text { laboratórios remotos colaborativos. (2009) }\end{array}$ & de laboratórios em engenharia \\
\hline $\begin{array}{l}\text { Aprendizagem global para o desenvolvimento sustentável no ensino superior: } \\
\text { tendências recentes e uma crítica. (2009) }\end{array}$ & $\begin{array}{l}\begin{array}{l}\text { Aprendizagem global para } \\
\text { sustentável }\end{array} \\
\text { o desenvolvimento }\end{array}$ \\
\hline $\begin{array}{l}\text { O que precisa ser aprendido para a sustentabilidade? Uma comparação de competências } \\
\text { de ensino de engenharia de bacharelado em três universidades europeias. (2009) }\end{array}$ & $\begin{array}{l}\text { Teoria e prática - competências desenvolvidas e } \\
\text { competências desejáveis }\end{array}$ \\
\hline $\begin{array}{l}\text { Melhorando a tomada de decisão para a sustentabilidade: um estudo de caso da Nova } \\
\text { Zelândia (2009) }\end{array}$ & $\begin{array}{l}\text { Programa de treinamento desenvolvido pela } \\
\text { universidade para tomadores de decisão externos. }\end{array}$ \\
\hline $\begin{array}{l}\text { Tornando a sustentabilidade 'real': usando pesquisa de grupo para promover a educação } \\
\text { para o desenvolvimento sustentável. (2008) }\end{array}$ & aprendizagem experiencial e competência da ação \\
\hline $\begin{array}{l}\text { Competências profissionais dos futuros professores: Perspectiva de diferentes } \\
\text { avaliadores e contextos. (2008) }\end{array}$ & $\begin{array}{l}\text { desenvolvimento de competências profissionais por } \\
\text { meio programas de estudos enriquecidos com os } \\
\text { princípios do desenvolvimento sustentável }\end{array}$ \\
\hline $\begin{array}{l}\text { Educação para o desenvolvimento sustentável (essência, objetivos e etapas da } \\
\text { construção). (2006) }\end{array}$ & 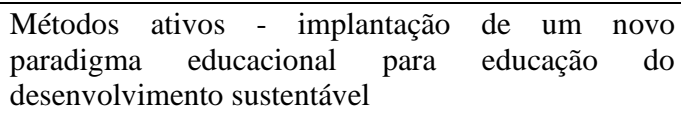 \\
\hline $\begin{array}{l}\text { Ensino superior para a sustentabilidade por meio de estudos de caso transdisciplinares: } \\
\text { uma abordagem inovadora para resolver problemas complexos do mundo real. (2006) }\end{array}$ & $\begin{array}{l}\text { Teoria e Prática - abordagem de estudo de caso } \\
\text { transdisciplinar como requisito no processo } \\
\text { educacional para o desenvolvimento sustentável }\end{array}$ \\
\hline Aprendendo a ser sistêmico: algumas reflexões de uma organização que aprende. (1996) & $\begin{array}{l}\text { Teoria e Prática - Abordagem do Sistema Crítico de } \\
\text { Aprendizagem }\end{array}$ \\
\hline
\end{tabular}

Fonte: Autores.

Os estudos levantados giram em torno da formação de professores universitários e o desenvolvimento de competências de sustentabilidade que fortaleçam o ensino e, portanto, a formação de alunos, ou seja de futuros professionais, com maior consciência da importância do desenvolvimento sustentável e que sejam agentes de transformação social. Neste sentido, em geral, enfatizam a necessidade de que todos os alunos e professores sejam capacitados e não apenas aqueles que se interessam de modo específico.

Um dos pontos mais relevantes observados é a integração da sustentabilidade nos currículos universitários, com destaque a graduandos, sobretudo dos cursos de engenharia. Os resultados também indicam uma crescente preocupação em alinhar inovação, metodologias ativas e mídias digitais no fomento e ensino do desenvolvimento sustentável. As pesquisas no campo do ensino voltam-se para analisar o que tem acontecido no campo acadêmico, enfocando a necessidade de construção de currículos sustentáveis. Contudo, existem lacunas no que tange a apresentação de propostas para estes currículos, assim como de modelos para programas de treinamentos de professores e, ainda, como sistematizar a avaliação destas possíveis mudanças. 
No ano de 2020 há um destaque quanto a adaptação no uso de tecnologias da informação. Talvez isso se deva ao direcionamento da educação presencial para modalidade "on line", ocorrida em razão da pandemia e consequente fechamento das Instituições de Ensino Superior. Este viés de ensino pode intensificar ao longo dos anos que virão, o que indica a necessidade de aprofundamento de pesquisas nesta área.

Alguns temas são frequentes nas pesquisas dirigidas ao ensino, tais como transdisciplinaridade e multidisciplinaridade, tendo em vista a consciência da necessidade do trabalho conjunto e colaborativo para o alcance do desenvolvimento sustentável em nível global. Cientes desta realidade, também foram identificados estudos ressaltando as redes de colaboração entre pesquisadores e importância de parcerias entre universidade e de universidades com empresas e com a comunidade.

\subsection{Análise das publicações na perspectiva da gestão e universidade como um todo}

A Gestão Sustentável no contexto das universidades ainda tem sido um campo pouco explorado pelos pesquisadores, ainda que estas instituições sejam reconhecidas como catalisadoras e atores-chave para o alcance do desenvolvimento sustentável. A questão é que a ênfase tem sido dirigida a educação. Os artigos identificados no mapeamento bibliométrico com ênfase na gestão são analisados a seguir:

\section{O desenvolvimento do corpo docente como um catalisador para a mudança curricular em direção à educação para} o desenvolvimento sustentável: uma perspectiva de produção (2012)

\section{Autoria: Matthias Barth e Marco Rieckmann}

A pesquisa descreve o caso de um programa de desenvolvimento de pessoal acadêmico, com foco no corpo docente, implementado por uma universidade equatoriana. Analisa em que medida esse programa tem efeitos positivos nas mudanças transformadoras em direção a uma universidade sustentável. O estudo de caso parte do pressuposto teórico de que a implementação da sustentabilidade no ensino superior e a aprendizagem social são impulsionadores da mudança organizacional.

Descreve o caso especifico de um programa de desenvolvimento de pessoal acadêmico, implementado na Universidad Técnica del Norte (UTN), Equador, em 2009 e que foi desenvolvido em conjunto com a projeto 'Ensino e Aprendizagem da Sustentabilidade' realizado pela UTN e pela Leuphana Universität Lüneburg, Alemanha. A pesquisa busca averiguar como se dá a aprendizagem e em que medida altera o desenvolvimento da organização. A partir daí analisa até que ponto esse programa tem efeitos positivos nas mudanças transformadoras em direção a uma universidade sustentável como um todo.

Os resultados do programa apresentaram desenvolvimento de competências individuais como resultado do processo de aprendizagem, mudanças no desempenho profissional que afetaram as práticas de ensino e impactos na organização como resultado de um processo de aprendizagem social.

Quanto a mudanças organizacionais, os participantes colaboraram em três medidas: (1) discussão dos objetivos do programa com os colegas, o que facilitou o processo de disseminação da ideia de sustentabilidade na universidade (2) influência nos processos de aprendizagem em sala e nos projetos de tese e (3) melhoria das suas habilidades interdisciplinares e aumento das oportunidades de colaboração. Além do que, no exercício de cargos ou funções oficiais, tornaram-se facilitadores da mudança organizacional, seja publicando informações relacionadas à sustentabilidade em um jornal da universidade ou incluindo a sustentabilidade nas políticas e estratégias do corpo docente.

Por fim, quatro aspectos destacaram-se nos resultados da pesquisa: interação entre teoria e prática, interdisciplinaridade, aprendizagem informal e vínculo das abordagens de liderança. 


\section{Universidades como atores potenciais para o desenvolvimento sustentável (2014)}

Autoria: Michael von Hauff e Thuan Nguyen

A pesquisa corresponde ao estudo sobre desenvolvimento sustentável em nível universitário na Alemanha, onde a implementação do desenvolvimento, em comparação com os esforços em outros países, foi considerada pelos autores como estando ainda está nos estágios iniciais, tendo em vista as três dimensões: econômica, social e ambiental.

Explana as Iniciativas e redes internacionais de desenvolvimento sustentável na área de educação superior e aborda os principais requisitos para uma universidade sustentável, aplicáveis as áreas de ações, modelos, estratégias, base organizacional, pesquisa, educação, operações, extensão. Por fim, analise os sistemas internacionais de avaliação de sustentabilidade para universidades, com foco em um estudo dirigido a experiencia alemã e com objetivo de acelerar os processos de transformação no contexto universitário.

Os resultados apresentados são provenientes de um projeto de pesquisa que inclui tanto a revisão e análise das atividades atuais da universidade relacionadas ao desenvolvimento sustentável, como também a construção de uma estratégia de sustentabilidade. Neste sentido, apresenta uma metodologia como ferramenta de gestão para servir as universidades em seus esforços na implementação da sustentabilidade em diferentes campos de ação.

Ao fim da pesquisa, os autores concluem que os sistemas de avaliação permitem que as universidades usem sistematicamente seu potencial de ação para desenvolvimento sustentável, iniciando, avaliando e acelerando o processo de sustentabilidade. Indica, ainda, a necessidade de integração das diferentes áreas, de envolver os stakeholders e, ainda, de incluir a sustentabilidade na declaração de missão ou no plano de desenvolvimento estratégico para toda a universidade.

\section{A abordagem de toda a instituição na Universidade de Tübingen: o desenvolvimento sustentável definido na} prática. (2020)

Autoria: Kerstin Schopp, Matthias Bornemann e Thomas Potthast

Este estudo de caso, dirigido a Universidade de Tübingen, na Alemanha, foi realizado na perspectiva da instituição como um todo, a partir de análise documental e investigação empírica sobre desenvolvimento de competências de sustentabilidade e sua aplicação prática. A pesquisa busca entender qual é a compreensão de desenvolvimento de sustentabilidade dos atores que estão envolvidos nos diferentes campus da Universidade de Tübingen, como e onde o desenvolvimento sustentável deve ser implementado e, ainda, que tipo de conhecimento e competências estes atores precisam para implementação nas áreas de operações, pesquisa, governança, transferência, ensino e educação e relatórios de sustentabilidade.

Primeiramente, descreve o Centro de Competência da Universidade e mostra como ele foi constituído. Segundo os realizadores do estudo de caso, o Centro de Competência é o ponto de conexão da rede, que reúne pessoas, conhecimentos e competências com foco no desenvolvimento sustentável no âmbito da universidade. É responsável por dispor da estrutura necessária para os projetos de desenvolvimento sustentável em todos os níveis universitários e captar as ideias das pessoas.

Apresenta, primeiramente o status quo das ações e da institucionalização do DS na Universidade de Tübingen. Em segundo lugar, apresentamos os resultados dos questionários e demonstramos como eles estão conectados ao entendimento de Brundtland sobre DS. Por fim, explicamos quais conhecimentos e competências precisam os atores envolvidos na promoção do DS na Universidade de Tübingen, de acordo com nossos entrevistados.

Descreve os entendimentos de desenvolvimento sustentável na Universidade de Tübingen, os valores por trás do desenvolvimento sustentável, e a percepção quanto ao conceito de DS de Brundtland. Como uma das principais contribuições 
do estudo explica quais conhecimentos e competências precisam os atores envolvidos na promoção do DS na Universidade de Tübingen, de acordo com aqueles que foram entrevistados. Por fim, são sugeridas opções e oportunidades de transferência dos resultados da pesquisa para outras Instituições de Ensino Superior em nível internacional.

4. Trabalhando com as divisões: dois eixos críticos no desenvolvimento de práticas profissionais transformadoras (2017)

Autoria: Helen Avery e Birgitta Nordén

O objetivo do artigo é fornecer um mapa conceitual de como mediar teoria e prática da sustentabilidade no ensino superior, tendo como base o campo acadêmico e a universidade como um todo. No contexto da ação, discute as maneiras pelas quais podem ocorrer a interação e mediação em ambientes de ensino superior, para conectar a pesquisa em sustentabilidade com programas vocacionais. Consiste, portanto, em uma análise do que a literatura dispõe sobre o tema e que permite observar diferentes formas de diálogo, a interação e fluxos de ideias contextualizadas a prática da sustentabilidade no âmbito das instituições de ensino superior.

Busca identificar as barreiras estruturais e os facilitadores ligados a pesquisa e o ensino orientados a ação, nas estruturas do ensino superior. Faz isso a partir de dois eixos críticos: conexões interambientais e espaços de reflexão transdisciplinar e integradora. De modo mais específico corresponde a uma discussão crítica de como as questões apresentadas se interconectam no contexto de ensino superior sueco, partindo do relatório de Educadores Universitários para o Desenvolvimento Sustentável.

O mapeamento conceitual aborda os seguintes temas: Sustentabilidade e aprendizado para a ação, ambientes de ensino superior orientados para a disciplina e para o profissional, incentivos e fragmentação, divisão entre teoria e prática, direção de cima para baixo, desenvolvimento participativo, mediação de questões de sustentabilidade no ensino superior, liderança e engajamento, flexibilidade e continuidade, Abordagens interdisciplinares e colaboração interuniversitária e, por fim, desenvolvimento de toda instituição para sustentabilidade.

A partir da análise dos artigos apresentados nesta sessão, é possível perceber a importância de dirigir novos estudos focados na gestão universitária sustentável, por serem o ambiente onde o ensino e pesquisa são desenvolvidos e em razão da força que exercem sobre a sociedade. O desenvolvimento sustentável precisa estar vinculado a todos os processos executados na instituição, em todos os níveis.

\section{Considerações Finais}

No decurso dos anos tem crescido, em nível global, o interesse pelo papel das universidades na implantação do desenvolvimento sustentável por meio da formação de profissionais capacitados a empreender estratégias de sustentabilidade. A literatura conta com pesquisas voltadas para o campo acadêmico, cujo objetivo é fomentar o desenvolvimento de competências nos estudantes e professores universitários a partir da reconfiguração dos currículos de graduação, construção de novas abordagens pedagógicas e metodologias inovadoras de aprendizagem, como os métodos ativos e a participação dos alunos na construção de um novo paradigma educacional. Este tem sido a principal ênfase nos estudos realizados até então. Foram identificados 82 artigos voltados a perspectiva da educação, enquanto que apenas 4 artigos apresentaram foco na gestão sustentável.

O mapeamento bibliométrico revelou que as pesquisas sobre universidade sustentável têm dirigido esforços em compreender quais competências são desejáveis para o desenvolvimento sustentável, quais são reais entre alunos e professores e qual a percepção destes em relação ao assunto. De modo mais amplo, é ascendente a preocupação com o vínculo entre teoria 
e prática. E, ainda, com o pensamento sistêmico e a interdisciplinaridade no realinhamento desta nova modalidade de educação no ensino superior.

Os resultados demonstram um latente interesse na inovação, incorporação de mídias digitais e promoção de habilidades empreendedoras nas novas práticas de ensino. É possível observar uma prevalência de estudos focados mais na análise do cenário atual das universidades em termos de desenvolvimento de competências de sustentabilidade. Existe uma lacuna na literatura quando se trata de propostas práticas ligadas a gestão universitária sustentável, englobando aspectos como operacionalização e sistematização de programas de treinamento voltados a implantação dos princípios do desenvolvimento sustentável no âmbito das instituições de ensino superior, além da apresentação de modelos de gestão sustentável e propostas de ferramentas de avaliação e acompanhamento no âmbito das universidades.

Como sugestão para futuras pesquisas, estudos dirigidos à qualificação de profissionais da educação com foco na sustentabilidade podem ser bastante representativos para o cenário atual, incluindo todos os níveis organizacionais, considerando a participação da liderança e dos diferentes stakeholders, e que possa contribuir com aspectos conceituais e metodológicos.

\section{Referências}

Avery, H. \& Nordén, B. (2017) Working with the divides Two critical axes in development for transformative professional practices - International Journal of Sustainability in Higher Education. 18(5), 666-680

Baker-Shelley, A, Zeijl-Rozema, A, \& Martens, P (2017). A conceptual synthesis of organisational transformation: How todiagnose, and navigate, pathways for sustainability at universities? Journal of Cleaner Production, 145, 262-276

Barth, M. \& Rieckmann, M. (2012) Academic staff development as a catalyst for curriculum change towards education for sustainable development: An output perspective - Journal of Cleaner Production. 26, 28-36

Bastida, M., García, A. V., Márquez, M. C., \& Blanco, A. O (2020). Fostering the sustainable development goals from an ecosystemconducive to the SE: The Galician's case. Sustainability 12(2), 1-17

Berchin at al (2018). The importance of international conferences on sustainable development as higher education institutions' strategies to promote sustainability: A case study in Brazil. Journal of Cleaner Production, 171, 756 - 772

Brundiers, K., Wiek, A. \& Redman, C. L. (2010). Real-world learning opportunities in sustainability: from classroom into the real world - International Journal of Sustainability in Higher Education. 11(4), 308-324

Di Nauta, P., Iannuzzi, E., Milone, M., \& Nigro, C (2020). The Impact of the Sustainability Principles on the Strategic Planning and Reporting of Universities. An Exploratory Study on a Qualified Italian Sample. Sustainability, 12, 7269

Faria, P. M. (2016). Revisão sistemática da literatura: contributo para um novo paradigma investigativo, metodologia e procedimentos na área da educação, aplicação prática aos temas desenvolvimento profissional docente e inovação educativa com tecnologias digitais. Santo Tirso. Ed. Whitebooks

Hauff, M. V. \& Nguyen, T. (2014). Universities as Potential Actors for Sustainable Development-Sustainability, 6, 3043-3063

Le Boterf, G. (2003). Desenvolvendo a competência dos profissionais. (3a ed). Artmed.

Leal Filho, W. at al (2018). The role of transformation in learning and education for sustainability. Journal of Cleaner Production, 199. 286-295

Litre, G., Bursztyn, M., Simoni, J., \& Reis, R (2020). Achieving the Sustainable Development Goals through good enough governance. Agrociencia Uruguay, 24(2)

Lozano, R, Lukman, R, Lozano, F. J, Huisingh, D, \& Lambrechts, W (2013). Declarations for sustainability in higher education: becoming better leaders, through addressing the university system. Journal of Cleaner Production, 48, 10-19

Munck, L., Borim-de-Souza, R., Castro, A. L., \& Zagui, C (2011). Modelos de gestão de competências versus processo de validação. Um ponto cego? Revista de Administração. 46(2), 107-121

Ofei-Manu, P., \& Didham, R. J (2018). Identifying the factors for sustainability learning performance. Journal of Cleaner Production, v.198, p.1173-1184

Schopp, K., Bornemann, M. \& Potthast, T. (2020) The whole-institution approach at the University of Tubingen: Sustainable development set in practice Sustainability, 2020, 12(3).

Valencia, S. C. at al (2019). Adapting the Sustainable Development Goals and the New Urban Agenda to the city level: Initial reflections from a comparative. International Journal of Urban Sustainable Development. 11:1, 4-23

Velazquez, L., Munguia, N., Platt, A. \& Taddei, J. (2006). Sustainable university: what can be the matter? Journal of Cleaner Production 14, 810-819 
Research, Society and Development, v. 10, n. 13, e444101321430, 2021

(CC BY 4.0) | ISSN 2525-3409 | DOI: http://dx.doi.org/10.33448/rsd-v10i13.21430

Wals, A. E. J. (2014). Sustainability in higher education in the context of the UN DESD: a review of learning and institutionalization processes - Journal of Cleaner Production, 8 -15

WCED - World Commission on Environmnt and Development (1987) Our Comon Future - The Brundtland Report - Oxford, oxford University Press

Zarifian, P. (2008). Objetivo competência: por uma nova lógica. Atlas. 